\title{
Information Integration of Engineering Construction Project Based on Management Process
}

\author{
Cui Bo \\ State Key Laboratory of Hydraulic Engineering Simulation and Safety, Tianjin University \\ Tianjin, China 300072 \\ cuib@tju.edu.cn
}

Keywords: engineering construction project; workflow; management process; information integration

\begin{abstract}
In Chinese information field of engineering construction, the traditional information management is on the function-oriented and data-oriented base, and cannot provide comprehensive and complete process-oriented support in the process management. There is a big gap especially in the information integration management. Based on workflow technology, this paper studies the information integration of engineering construction project based on management process, and realizes the integration of management and organization and information systems integration of management process. The research results can provide the theory and method support for the information integration of engineering construction project.
\end{abstract}

\section{Introduction}

The implementation of modern construction engineering project is a complex system composed jointly by the multiple units and a number of departments; all participants have to standardize the information process between them and organize the reasonable information flow to achieve communication at any time. The management process is the bridge between the organization and the information management system. In the Chinese information field of engineering construction, the traditional management information system is on the function-oriented and data-oriented base, and cannot provide comprehensive and complete process-oriented support in the process management. There is a big gap especially in the information integration management. In recent years, some domestic and foreign scholars have used the workflow technology to realize supporting the management process through the project management information system in the engineering construction $[1,2,3]$. Based on workflow technology, this paper studies the information integration of engineering construction project based on management process.

Information integration of engineering construction project is to realize the sharing of data and information among the project participants between the various processes of the whole life cycle of the engineering construction project. Information integration is not a simple way to achieve information sharing between various departments on technology, but from the perspective of system operation to ensure that each part of the system can send the right information to people who need the information at the right time, place and in the right way at each stage of the operation. The construction project information integration based on the management process needs to solve two problems: the integration of management work and organization; information system integration of management process.

\section{Workflow management system and workflow modeling}

Workflow is a business process that can be fully or partially automatically operated, and it can be delivered and executed among different people according to a series of process rules, documents, information or tasks [4]. Workflow management is the method and technology for the optimization of the work process. It focuses on realizing the automation of the organization process using information 
and communication technology, and can provide strong technical support for CPR to better achieve the organizational objectives.

Workflow management system is a software system, which completes the definition and management of workflow and creates a workflow and manage its execution through software definition in accordance with the predefined workflow logic in the computer. It runs on one or more workflow engines, which explain the definition of processes to advance the execution of a workflow instance. A complete workflow system consists of two parts: The part of process modeling (i.e., workflow modeling) and the part of workflow engine (provide the environment for the operation of the model). The management business process after the reorganization is still a general work process, and needs to be transformed into a workflow suitable for computer processing. The completion of the project management process is achieved through the implementation of a number of specific jobs, these specific jobs are formed after the specific executive personnel (resources) and the relationship between activities (rules) are given by the activities and this process is known as workflow modeling.

There are various workflow modeling methods, and the modeling method with theoretical basis of Petri net model and its extension is widely used. The advantages of using Petri net for model the workflow modeling: Intuitive graphical representation; Include strict semantics and graphic languages; Petri net is a state-based modeling method; Explicit representation of states and events; Petri net has strong analytical techniques and means. Petri net is suitable for the workflow modeling of workflow management module of construction project management information system based on the management process.

\section{The integration of management work and organization}

A construction project has a lot of management work, management work is composed of a number of management activities, and management activities can be divided into a series of management processes; they interact with and influence each other and are of systematic hierarchy, aggregation, correlation and integrity. The management of the project can be divided from different angles, such as the process of engineering construction, management functions and the basic process of project management to get different breakdown structure MWBS of management work. The general principle of MWBS preparation should take the target system of project as the leading factor and is based on the project management behavior system and the total task of the project. The project management process should be decomposed through the system analysis method one by one from top to bottom, from rough to fine and from beginning to end until it is decomposed into many project management process units that are interrelated, mutually influenced and mutually dependent. These management process units are regarded as the objects of project management and their logical relationships are determined to make the management function activities to meet the requirements of design, planning, control and operation of project.

The integration of management work and organization is the integration of the decomposition structure MWBS of management work and the decomposition structure OBS of the organization. MWBS successively decomposes the management work to the lowest level of management process units, OBS decomposes the project organization so that a single working group can be determined, and each manager of these groups is responsible for the corresponding management process unit. MWBS and OBS can realize that the management working tasks can correspond and adapt to the responsible person in the respective lowest level, so that MWBS, OBS and project management process unit constitute a public framework for project management system. Project management process unit, as the basic center of the framework, collects, analyzes information, evaluates the implementation situation and submits a report. Under this public framework, realize the integration of all management information, such as management work, organization, responsibility and plan, control of various levels administrative personnel, which is the integration of management work and organization.

In practice, the integration of OBS and MWBS should be resolved by the establishment of the corresponding OBS to the corresponding MWBS. First, according to MWBS, starting from the lowest level of the project management process unit, set up the corresponding minimum work 
package and working group of OBS, then according to the properties and characteristics of the working group and the human resources situation of the internal organization, set up the upper level of functional departments corresponding to MWBS until the formation of the highest level of the OBS, so the organization is formed on the basis of the breakdown and integration of project management work.

\section{Information system integration of management process}

The goal of integration management of management process is, through the application of integrated management method, to realize the seamless connection of each management process to ensure the realization of the functional goal of the whole system. Therefore, the information system integration management of the management process mainly includes two parts: Control and manage the management process in the each subsystem, and realize the target of the process management; Deal with the interface between the management processes, comprehensively manage the whole management information system, and promote the realization of the general objective of the construction project.

\section{Integrated management method for management processes}

(1) The establishment of a unified standard encoding system

In the whole life cycle of construction project, in order to achieve all the management functions, it is necessary to design a variety of types of encoding systems, such as the progress encoding system, contract encoding system, equipment encoding system and document encoding system, which constitutes a complex encoding system. On the basis of the structured decomposition of the project management work, encode the management activity and the corresponding management process of each project. Respective encoding standards are adopted for the different functional subsystems to meet the needs of constructing management information system. As for the encoding system built on the basis of MWBS, each management function work can realize the information communication, eliminating obstacle of the process interface at the greatest extent.

(2) Establish process business database

Specify the design, purchase, construction and other specific process models under professional management from the view of standard system operation, organize database under professional design from the view of workflow, so as to straighten out the data relation among design, purchase, construction and other professional management works. Establish the integrated database including management database and engineering database which focus on project document information, form electronic document control system based on this, reduce the redundant data, and improve the applicability of system.

(3) Information standardization of interface between processes

When the management process is docked, the sufficient information communication is the key factor of docking. Interface information is managed by management information system, and the specific field and table are usually the information exchange tools across system interface. In the implementation of management activities, the document report formed should be standardized and systematized, the structure and content of report and table system are defined completely, and the format and data structure of statement are standardized.

(4) Optimize management process by using modern information technology

Realize cooperation for project management activities by using network technology, adjust the participants in relevant management works, optimize the implementation route, reduce the non-value-added activities sharply while reduce the process internal conflict brought by organizing interface in the implementation of reducing process, so as to improve the efficiency of management work. Realize data sharing by database and network technology, change the serial structure into parallel structure, strengthen cooperation degree in the implementation process of feedback structure, change the structure of process, reduce the process conflict and improve the process efficiency.

\section{Integrated modeling of management process}

Establish integrated model of management process by process modeling technology, deeply analyze the relation between processes, and be in favor of realizing information integration of each 
process. The full name of IDEF (ICAM Definition Method) is integrated computer aided manufacturing definition method, a set of system analysis and design method developed by ICAM engineering of United States Air Force in the late 1970s and early 1980s based on structured analysis and design method [5]. IDEF0 is one of the methods, which is used to describe the functional activity and process modeling of system.

At the beginning of modeling, IDEF0 method firstly defines each activity of system and is shown by IDEF0 node, it includes 5 basic elements: Functional box, input (I), output (O), and control (C) and mechanism (M). Functional box shows one activity and process task to be analyzed, and the arrow defines the constraint of functional box (I, C, O and M). IDEF0 method is used as the structured analysis method to model in the functional process of system, analyze the activity process of system, and take top-to-down gradually layered and refined structured modeling method for system process. IDEF0 figure can be shown by A0, A1, A2, ...., where A0 is father figure, A1, A2, .... are refined to be A11, A12, A13,.... and A21, A22, A23,..... respectively, and so on, so as to constitute the process model of the overall system. Figure 1 shows the hierarchical breakdown structure of IDEF0 model in the construction project management process.

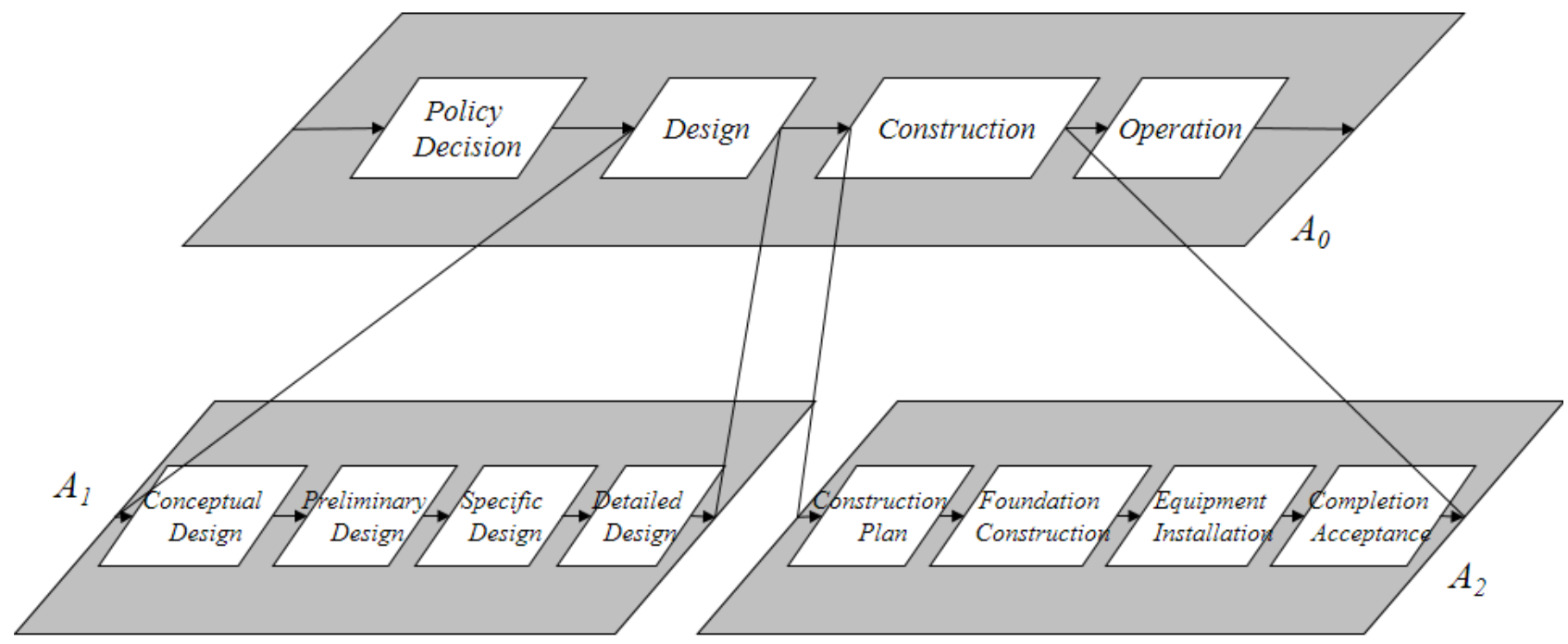

Fig. 1 the hierarchical breakdown structure of IDEF0 model in the construction project management process

\section{Integrated framework of management work based on workflow}

PMBOK divides the project management process into five process groups: Start, plan, implement, control and end. The process group is correlated by the results created by them, and the output or result of each process group becomes the input of next process group. In the middle process group, these relations are conducted repeatedly. For one specific project, each stage of the project includes several specific works, and these works are also considered as a new project derived, but they are managed according to 5 basic processes of project management. Although the schedule, investment, quality and other management works of construction project are different greatly in management object, manager, management content and management objective, each management work is conducted according to the management process composed by 5 basic processes strictly; the integration of project management and application can be realized based on workflow.

The integrated framework of management work based on workflow is one three-layer system structure used to realize construction project management application integration based on management process of workflow (as shown in Figure 2). Three-layer system structure includes management process layer, adapter layer and management application layer. Each management process workflow in the management process layer is composed of above 5 process groups, each process group shows the executing state of management work, and different management process workflows are conducted according to each management process model. In the framework, the information required by communication among workflow, management application and different management applications is converted by adapter. The information flow between workflow and 
management application as well as data compatibility between different applications are realized by adapter, so that each process group can establish relation with a series of management application. Hereafter, the executing process of management application and data flow are controlled by workflow, and the detail property of flow state is separated to be processed by management process model, which can improve the efficiency and stability of system.

The adapter is composed of three interfaces and one data converter (as shown in Figure 6). The input interface is used to receive the data from management application, and the output interface is used to output data to management application. The data converter between two interfaces is used to convert the input data into the format required by output data. The workflow interface is used to exchange the control data between management application and workflow. Due to existence of adapter, the modification on each management application subsystem is reduced when the management application integration of process is executed.

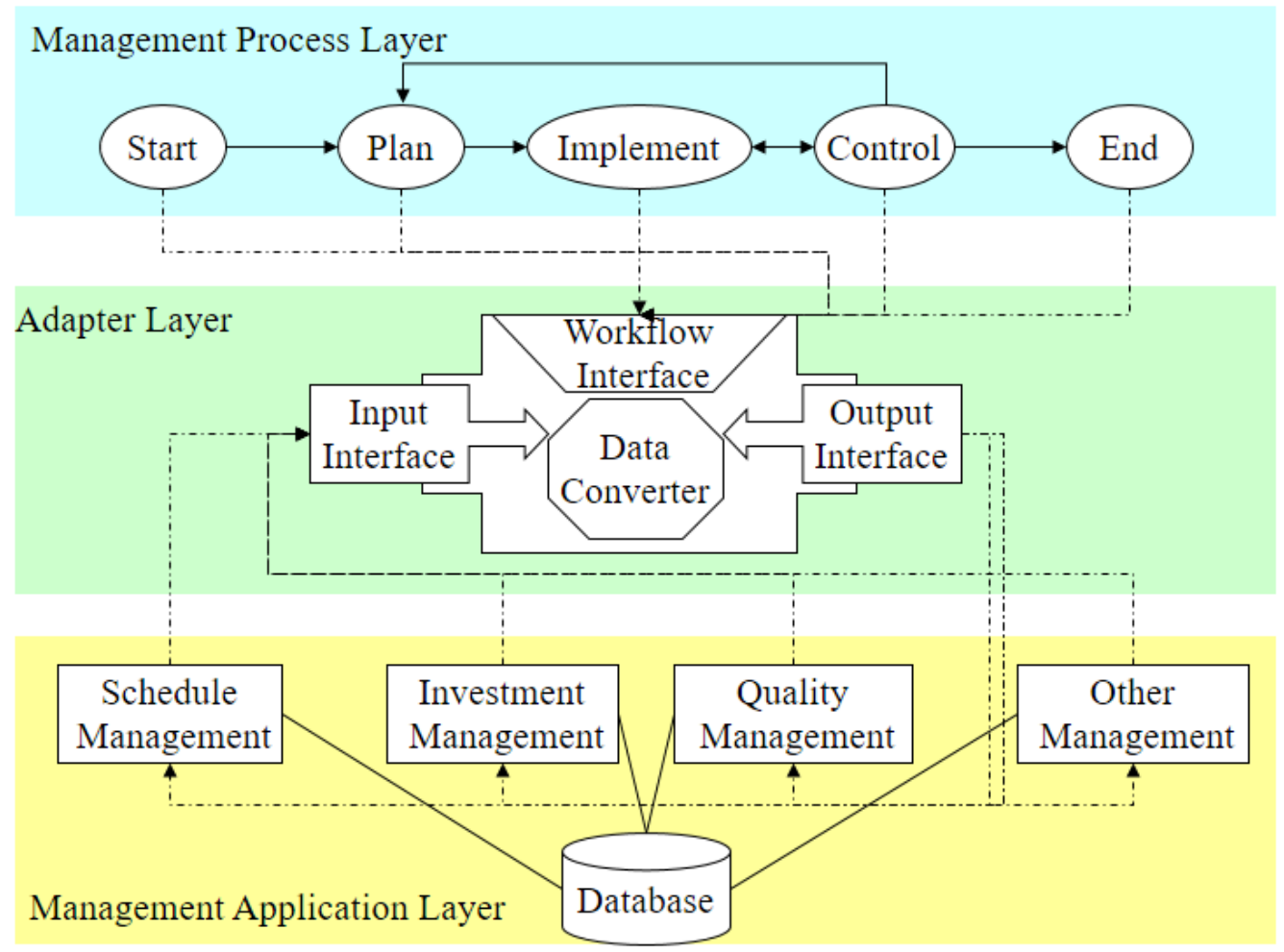

Fig. 2 the integrated framework of management work based on workflow

\section{Summary}

Based on workflow technology, this paper studies the information integration of engineering construction project based on management process. On one hand, realize integration of management work and organization by researching the integration method of management work breakdown structure (MWBS) and organization breakdown structure (OBS). On the other hand, research the integrated management method of management process, apply IDEF0 method for integrated modeling of management process, and build integrated framework of management work based on workflow, so as to realize the integration of information system manner of management process. The research results can provide the theory and method support for the information integration of engineering construction project.

\section{Acknowledgements}

This work is financially supported by the National Natural Science Foundation of China (No. 51209159) (No. 51379006) and the National Basic Research Program of China (973 Program) (No. 2013CB035906). 


\section{References}

[1] Fang Yongquan, Wang Xuben. Scientific and Technological Management of Land and Resources, 2002, 4 (13)

[2] Dong-Eun Lee, Jonathan J.Shi. Construction research congress, ASCE, San Diego, USA, 2005.4

[3] Uwe R, Thomas K. Http://e-pub.uni-weimar.de/volltexte/2004/170/pdf/icccbe-x_159.pdf

[4] Luo Haibin, Fan Yushun, Wu Cheng. Journal of Software, 2005, 3 (3)

[5] Chen Yuliu : IDEF Modeling Analysis and Design Method (Trans Tech Publications, Switzerland 1987). 